See discussions, stats, and author profiles for this publication at: https://www.researchgate.net/publication/333340334

\title{
Site-selective integration of MoS2 flakes on nanopores by means of electrophoretic deposition
}

Article - May 2019

Dol: 10.1021/acsomega.9b00965

CITATIONS

10 authors, including:

Dario Mosconi

University of Padova

14 PUBLICATIONS 102 CITATIONS

SEE PROFILE

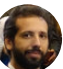

Andrea Jacassi

Istituto Italiano di Tecnologia

21 PUBLICATIONS 59 CITATIONS

SEE PROFILE
READS

27

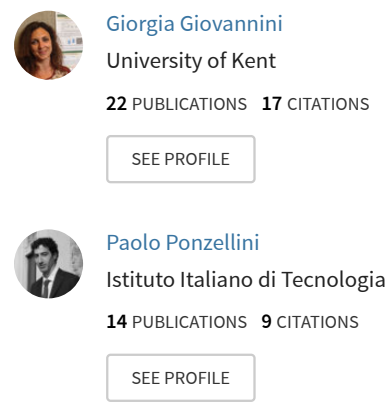

Some of the authors of this publication are also working on these related projects:

cell mechanics View project

Terahertz technology View project 


\title{
Site-Selective Integration of $\mathrm{MoS}_{2}$ Flakes on Nanopores by Means of Electrophoretic Deposition
}

\author{
Dario Mosconi, ${ }^{\dagger}$ Giorgia Giovannini, ${ }^{\ddagger}$ Andrea Jacassi, ${ }^{\ddagger}$ Paolo Ponzellini, ${ }^{\ddagger}$ Nicolò Maccaferri, ${ }^{\ddagger}, \perp$ \\ Paolo Vavassori, ${ }^{\S}$ Michele Serri, ${ }^{\ddagger 0}$ Michele Dipalo, ${ }^{\ddagger 0}$ Daniel Darvill, ${ }^{\ddagger}$ Francesco De Angelis,

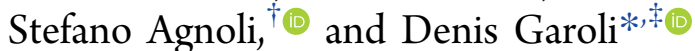 \\ ${ }^{\dagger}$ Dipartimento di Chimica, Università degli Studi di Padova, Via Marzolo 1, 35131 Padova, Italy \\ ${ }^{\ddagger}$ Istituto Italiano di Tecnologia, Via Morego, 30, I-16163 Genova, Italy \\ ${ }^{\S}$ CIC nanoGUNE, Tolosa Hiribidea, 76, E-20018 Donostia-San Sebastian, Spain \\ ${ }^{\perp}$ Physics and Materials Science Research Unit, University of Luxembourg, L-1511 Luxembourg, Luxembourg
}

Supporting Information

ABSTRACT: Here, we propose an easy method for siteselective deposition of two-dimensional (2D) material flakes onto nanoholes by means of electrophoretic deposition. This method can be applied to both simple flat nanostructures and complex three-dimensional structures incorporating nanoholes. The deposition method is here used for the decoration of large ordered arrays of plasmonic structures with either a single or few layers of $\mathrm{MoS}_{2}$. In principle, the plasmonic field generated by the nanohole can significantly interact with the 2D layer leading to enhanced light-material interaction. This makes our platform an ideal system for hybrid 2D material/ plasmonic investigations. The engineered deposition of $2 \mathrm{D}$

materials on plasmonic nanostructures is useful for several important applications such as enhanced light emission, strong coupling, hot-electron generation, and 2D material sensors.

\section{INTRODUCTION}

In the last decade, there has been an increasing interest in the investigation of two-dimensional (2D) materials, such as graphene and various transition metal chalcogenides. ${ }^{1-5}$ Molybdenum disulfide $\left(\mathrm{MoS}_{2}\right)$, in particular, which consists of S-Mo-S layers bonded by van der Waals interactions is noted. $\mathrm{MoS}_{2}$ offers a powerful platform for applications in nanophotonics and optoelectronics due to its remarkable optical properties. ${ }^{6,7}$ Moreover, monolayer $\mathrm{MoS}_{2}$ can also be integrated with other low-dimensional materials such as quantum dots, ${ }^{8}$ nanowires, ${ }^{9,10}$ and other $2 \mathrm{D}$ materials ${ }^{11,12}$ to form hybrid nanostructures with intriguing electronic and optical properties.

Regarding the latter, extensive interest from researchers has been focused in the last few years on exploring the potential of these physical phenomena, especially light-matter interactions, such as multiexciton photoluminescence, interlayer exciton coupling, strong coupling, and the valley polarization effect. $^{13-17}$ However, controlling the optical properties of a $\mathrm{MoS}_{2}$ layer(s) is still a great challenge and significant efforts are still required. The active control of $\mathrm{MoS}_{2}$ can be, in principle, achieved by utilizing photonic and plasmonic nanostructures. $^{18-23}$ However, one of the main limitations of $\mathrm{MoS}_{2}$ application in photonics and plasmonics is related to the challenge of aligning nanostructures with the $2 \mathrm{D}$ material. New

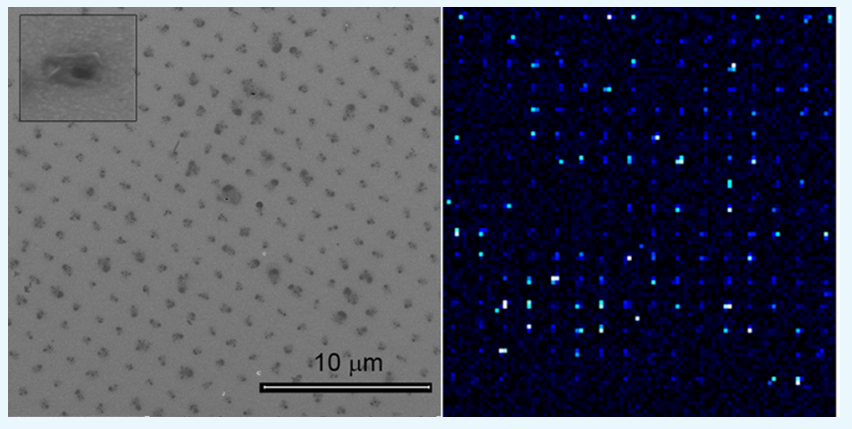
approaches for easy and controlled integration of 2D materials with metallic or semiconductor nanostructures are now of great importance in various fields of research. We have recently proposed and demonstrated the possibility to control the deposition of single-layer $\mathrm{MoS}_{2}$ flakes on metallic nanostructures by means of chemical conjugation. ${ }^{24}$ Here, we propose an alternative approach that we demonstrate can be applied to any substrates comprising nanoapertures, decorating them with $\mathrm{MoS}_{2}$ flakes as either a single or few layers. As we will show, the procedure is based on electrophoretic deposition (EPD) where the substrate does not act as an electrode. This method allows for high yield deposition onto both flat nanostructures and three-dimensional (3D) elements.

We surmise that the structures that can be easily prepared with this method can find several interesting applications in all of the present fields of research in which 2D materials are the core. Such examples include: enhanced light emission, ${ }^{13,21,25-30}$ strong coupling from plasmonic hybrid structures, $^{17}$ hot-electron generation, ${ }^{31,32}$ and $2 \mathrm{D}$ material sensors in general. ${ }^{33-38}$

Received: April 4, 2019

Accepted: May 17, 2019

Published: May 29, 2019 


\section{RESULTS AND DISCUSSION}

An illustration of the deposition process can be found in Figure 1 (top panel). A microfluidic chamber containing two platinum
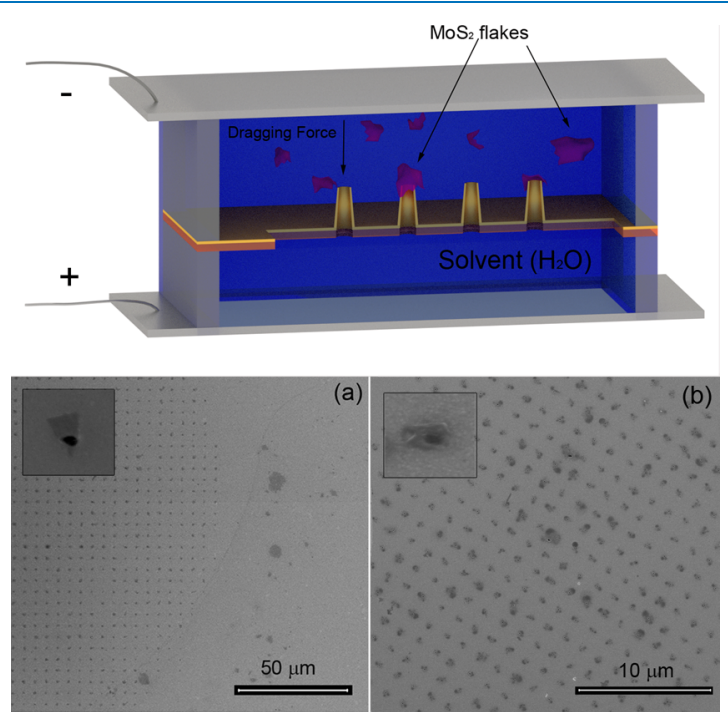

Figure 1. Top panel: illustration of the electrophoretic process; (a) scanning electron microscopy (SEM) micrographs of gold nanohole arrays decorated with $\mathrm{MoS}_{2}$ flakes by means of EPD (2 V-5 min); (b) EPD with full coverage ( $15 \mathrm{~V}-5 \mathrm{~min})$. Insets: details of deposited flakes.

(Pt) electrodes at the anode and cathode sides has been used to perform the EPD (see Supporting Information SI\#1). The chamber has been designed to place a $1 \times 1 \mathrm{~cm}^{2}$ sample that acts as a separation membrane between the two sides. The distance between the two electrodes (designed with a size of 1 $\times 1 \mathrm{~cm}^{2}$ ) is about $5 \mathrm{~mm} . \mathrm{MoS}_{2}$ flakes obtained from a chemical exfoliation process ${ }^{39-41}$ (see Methods) and suspended in $\mathrm{H}_{2} \mathrm{O}$ are injected through a microchannel in the cathode side, whilst the anode side is filled with the solvent (in our case $\mathrm{H}_{2} \mathrm{O}$ ).

The EPD can be performed, thanks to the non-zero electrophoretic mobility $\left(\mu_{\mathrm{e}}\right)$ of the $\mathrm{MoS}_{2}$ in $\left(\mathrm{H}_{2} \mathrm{O}\right)$ solution. The $\mu_{\mathrm{e}}$ of $\mathrm{MoS}_{2}$ flakes measured by dynamic light scattering (DLS; Malvern Zetasizer nano ZS) is $-3.10 \pm 0.03 \mu \mathrm{mcm} / \mathrm{Vs}$ (Table 1). In particular, the software measures the electro-

Table 1. $\zeta$-Potential and Electrophoretic Mobility of the $\mathrm{MoS}_{2}$ Flakes Measured by DLS in Water ${ }^{a}$

$\begin{array}{lc} & \text { DI water } \\ \zeta \text {-potential }(\mathrm{mV}) & -39.57 \pm 0.38 \\ \text { electrophoretic mobility }(\mu \mathrm{mcm} / \mathrm{Vs}) & -3.10 \pm 0.03\end{array}$

${ }^{a}$ Values are reported as an average of three measurements $(n=3) \pm$ SD.

phoretic mobility of the flakes and by means of Henry's equation the values of $\zeta$ ( $\zeta$-potential) are calculated considering parameters related to the environmental conditions such as the dielectric constant $(\varepsilon)$, viscosity $(\eta)$ of the solvent, and Henry's function $(f(k a))$

$$
\mu_{\mathrm{e}}=\frac{2 \varepsilon \zeta}{3 \eta} f(k a)
$$

Once the charged $\mathrm{MoS}_{2}$ flakes are dispersed in water, ions of the opposite charge will be absorbed at the surface forming a layer of strongly adhered ions (Stern layer), which becomes a diffuse and dynamic layer comprised of a mixture of ions with the increasing distance from the surface. Until a certain distance from the surface, these ions will move with the particle (slipping plane). The $\zeta$ measured is related to the potential existing between the surface and the slipping plane. Both Stern and diffuse layers form the electric double layer (EDL), which determines the electrical mobility of the flakes in suspension under an electric field measured by DLS. ${ }^{57}$ Several studies can be found in the literature where EPD is achieved in organic solvent, ${ }^{39}$ but to achieve an effective and efficient EPD additives are often added to the nonaqueous solvent. ${ }^{40,41}$ As $\mathrm{MoS}_{2}$ flakes are found to be stable in water, this avoids unnecessary complexity in altering the organic solution when the material can just be diluted in aqueous solvents.

EPD has been already proposed for $\mathrm{MoS}_{2}$ nanosheet deposition for several applications. ${ }^{42-44}$ With respect to the previously reported cases, here, the substrate where the flakes are deposited is not used as an electrode. In contrast, the nanoholes present in the substrate allow for the through-flow of ions, whilst also representing a barrier to the $\mathrm{MoS}_{2}$ flakes, which cannot pass due to their size and are deposited. An illustration of the setup for EPD can be found in Figure 1 (top panel) and a summation of the stages of the process are given here. The nanohole array prepared on a $\mathrm{Si}_{3} \mathrm{~N}_{4}$ membrane (see Methods) is first cleaned in oxygen plasma for $60 \mathrm{~s}$ to facilitate the deposition. The sample in the ground state is placed in the microfluidic chamber and the chamber sides are filled with $\mathrm{MoS}_{2}$ suspended in $\mathrm{H}_{2} \mathrm{O}$ and distilled $\mathrm{H}_{2} \mathrm{O}$, respectively. A suitable voltage for the required deposition thickness is then applied for $5 \mathrm{~min}$ to allow for electrophoretic deposition. The chamber is then opened, and the deposited final sample is rinsed with EtOH. Theoretically, this method can be used on any nanostructure comprising a nanohole as we have verified here on planar and 3D metallic nanohole arrays as well as dielectric $\left(\mathrm{Si}_{3} \mathrm{~N}_{4}\right)$ nanoholes. In the case of large rectangular arrays of metallic nanoholes, the substrate is a $\mathrm{Si}_{3} \mathrm{~N}_{4}$ membrane coated with a $100 \mathrm{~nm}$ thick gold film, where holes of $100 \mathrm{~nm}$ in diameter were prepared by means of focused ion beam (FIB) lithography.

To compare the effectiveness of EPD with a simple drop cast deposition, we dropped $20 \mu \mathrm{L}$ of $\mathrm{MoS}_{2}$ in $\mathrm{H}_{2} \mathrm{O}$ on a gold nanohole substrate and let it dry, with a final rinse in $\mathrm{EtOH}$. As expected (see SI\#2), the resulting substrate was found to be randomly coated, though a large number of holes are decorated with flakes. In our first EPD experiments, we observed that site-selective deposition is possible using a relatively low applied voltage $(2 \mathrm{~V})$. Nevertheless, this did not lead to $100 \%$ coverage of the nanoholes array and to low reproducibility of the process (as illustrated in Figure 1a). We considered $\mathrm{MoS}_{2}$ degradation as a potential cause of the low reproducibility and checked by performing different $\zeta$-potential measurements simulating aging effects (see SI\#3). To improve the \% of coverage to include all of the nanoholes in the array, we performed EPD while increasing the applied voltage up to 15 V. Figure 1a,b illustrate how, in this way, a full coverage could be easily obtained.

Once we demonstrated the proof-of-concept for controlled decoration of nanohole arrays, we investigated the applicability of this method to the case of $3 \mathrm{D}$ structures (Figure $2 \mathrm{a}-\mathrm{d}$ ). The fabrication of 3D plasmonic structures comprising a nanochannel/nanohole has been shown for different designs and materials in several recent papers. ${ }^{45-47}$ The main advantage of 


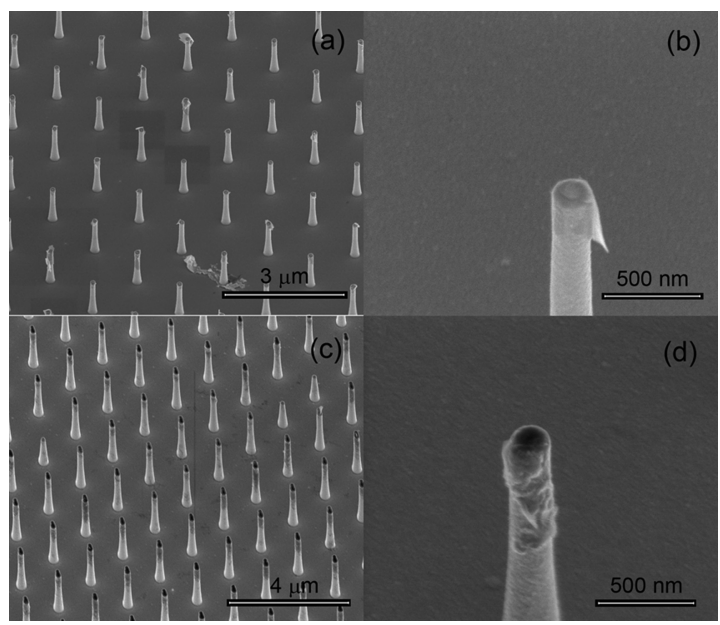

Figure 2. SEM micrographs of $3 \mathrm{D}$ antennas decorated with $\mathrm{MoS}_{2}$ flakes by means of EPD. (a, b) $15 \mathrm{~V}$ applied voltage, $5 \mathrm{~min}$; (c, d) 25 $\mathrm{V}$ applied voltage, $5 \mathrm{~min}$; (b, d) details of deposited flakes.

these particular structures is the possibility to confine and enhance the electromagnetic field (e.m.) in a narrow gap at the apex of the structure. With respect to a nanohole on a flat metallic film, the e.m. field enhancement can be significantly higher and the integrated nanochannel can also act as a nanofluidic element. ${ }^{24}$

Examples of electrophoretic deposition of $\mathrm{MoS}_{2}$ flakes over these substrates are reported in Figure 2. As has already been stated, the vertical antennas using a 3D design can be prepared with different aspect-ratio: within the range of a few hundreds of nm high up to a few $\mu \mathrm{m}$. We expect that higher antennas (see SI\#4) introduce a very high resistivity in the ion flux and, in fact, it was not possible to obtain a reproducible electrophoretic deposition at voltages up to $15 \mathrm{~V}$. By decreasing the vertical dimension down to $1 \mu \mathrm{m}$ and increasing the diameter of the nanochannel (see Methods for details), it was possible to obtain the ordered deposition. Actually, by increasing the voltage up to $25 \mathrm{~V}$, a full coverage deposition with high reproducibility was achievable. Figure $2 a-c$ illustrate some examples of EPD performed onto 3D antennas at different applied voltages. A drawback related to the high applied voltage used for EPD onto 3D structures is the deposition of many flakes also around the 3D antennas. In all of the cases, the deposition results in a partially covering layer that crinkles around the metal in many ways, ranging from small flakes covering only the top hole to large flakes wrapping around the 3D body of the structure. Before discussing how we evaluated the thickness (in terms of the number of layers) of the deposited flakes, we want to underline that our EPD method does not necessarily require a metallic substrate, but can also be applied to nonconductive substrates patterned with nanoholes. A demonstration of EPD on a dielectric substrate is reported (see SI\#5) where we used a patterned $\mathrm{Si}_{3} \mathrm{~N}_{4}$ membrane to decorate nanoholes with $\mathrm{MoS}_{2}$ flakes.

A well-known method ${ }^{7,48-51}$ to measure the thickness, in terms of the number of layers, of a $\mathrm{MoS}_{2}$ flake is based on Raman spectroscopy. $\mathrm{MoS}_{2}$ presents two main Raman modes in the shifts ranging between 360 and $420 \mathrm{~cm}^{-1}$, i.e., the inplane $\left(\mathrm{E}_{2 \mathrm{~g}}{ }^{1}\right.$ at $\left.\sim 380 \mathrm{~cm}^{-1}\right)$ and out-of-plane $\left(\mathrm{A}_{\mathrm{gg}}\right.$ at $\sim 404$ $\mathrm{cm}^{-1}$ ) modes. The $\Delta f$ (spectral distance) between these two modes is known to steadily increase with the number of layers. A value below $20 \mathrm{~cm}^{-1}$ is typically associated with a single layer, whereas the values between 20 and $23 \mathrm{~cm}^{-1}$ are associated with bi-layer flakes and higher values correspond to 3 or more deposited layers. Here, we used this procedure (already reported in one of our recent papers ${ }^{24}$ ) to verify the deposition of $\mathrm{MoS}_{2}$ flakes and to evaluate the number of layers deposited over the different positions. Raman spectroscopy measurements were performed using a Renishaw InVia Microscope Raman system with a $50 \times 0.95$ NA objective, collecting the signal with a spectral resolution of $2.5 \mathrm{~cm}^{-1}$ and an integration time of $1 \mathrm{~s}$. We used an excitation wavelength of $532 \mathrm{~nm}$ and the intensity of the standard peak at $520 \mathrm{~cm}^{-1}$ from a silicon substrate was used to calibrate the system. The results of our measurements can be found in Figure 3. Figure

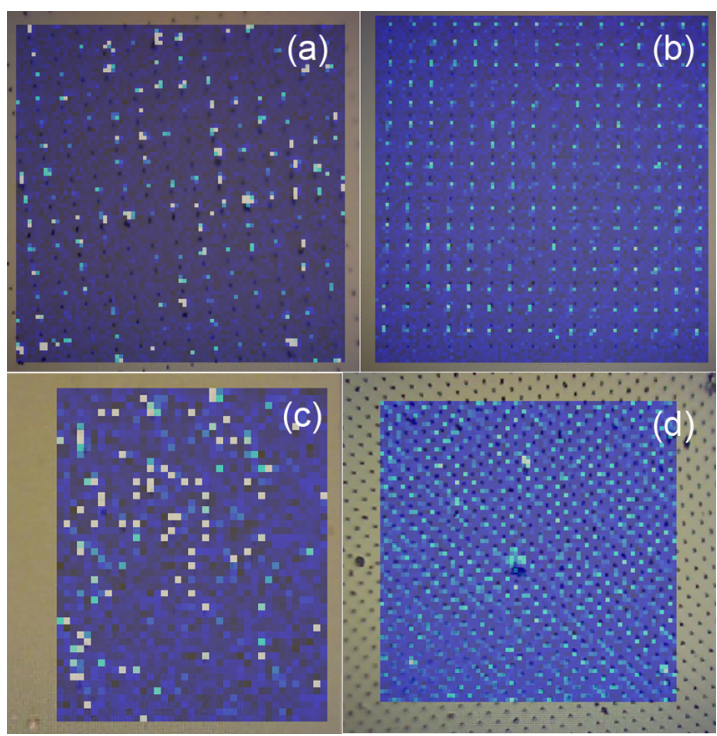

Figure 3. Raman maps reporting shifts between 400 and $410 \mathrm{~cm}^{-1}$ (in correspondence of $\mathrm{A}_{1 \mathrm{~g}}$ Raman mode). (a) EPD onto planar nanoholes $(2 \mathrm{~V})$; (b) EPD onto nanoholes (15 V); (c) EPD onto 3D antennas $(15 \mathrm{~V})$; and $(\mathrm{d})$ EPD onto 3D antennas $(25 \mathrm{~V})$.

3a,b show the map over large arrays of metallic nanoholes for low $(2 \mathrm{~V})$ and high $(15 \mathrm{~V})$ applied voltage, respectively. Figure $3 \mathrm{c}, \mathrm{d}$ refer to measurements performed on $3 \mathrm{D}$ antennas at 15 and $25 \mathrm{~V}$, respectively. Raman shifts integrated counts in the range between 400 and $410 \mathrm{~cm}^{-1}$ (in correspondence to the $A_{1 g}$ Raman mode) have been used to evaluate the coverage of the $\mathrm{MoS}_{2}$ over the maps. As verified by SEM investigations, a significant difference appears amongst the samples. In all cases, full site-selective deposition over the nanohole array has been achieved once a potential of 15 and $25 \mathrm{~V}$ has been applied to flat nanoholes and 3D antennas, respectively. For lower applied voltages, the flakes' deposition is still verified, but not all of the arrays are covered. The Raman maps not only demonstrate the actual site-selective EPD, but they can also be used to perform statistical analysis of the thickness of the deposited flakes.

Figure $4 \mathrm{a}, \mathrm{b}$ report statistical analysis of over about 200 and 100 different measured points of the Raman spectra for the metallic nanoholes and 3D nanoantenna samples deposited at 2 and $15 \mathrm{~V}$, respectively. As illustrated, the measured points were fit by Lorentzian functions (Figure 4c). The in-plane $\left(\mathrm{E}_{2 \mathrm{~g}}{ }^{1}\right.$ at $\left.\sim 380 \mathrm{~cm}^{-1}\right)$ and out-of-plane $\left(\mathrm{A}_{1 \mathrm{~g}}\right.$ at $\left.\sim 404 \mathrm{~cm}^{-1}\right)$ Raman modes were always clearly visible and used in analysis. The difference between the $\mathrm{E}_{2 \mathrm{~g}}{ }^{1}$ and $\mathrm{A}_{1 \mathrm{~g}}$ modes $(\Delta f)$ is known to steadily increase with the number of layers; ${ }^{7,48-51}$ hence, 

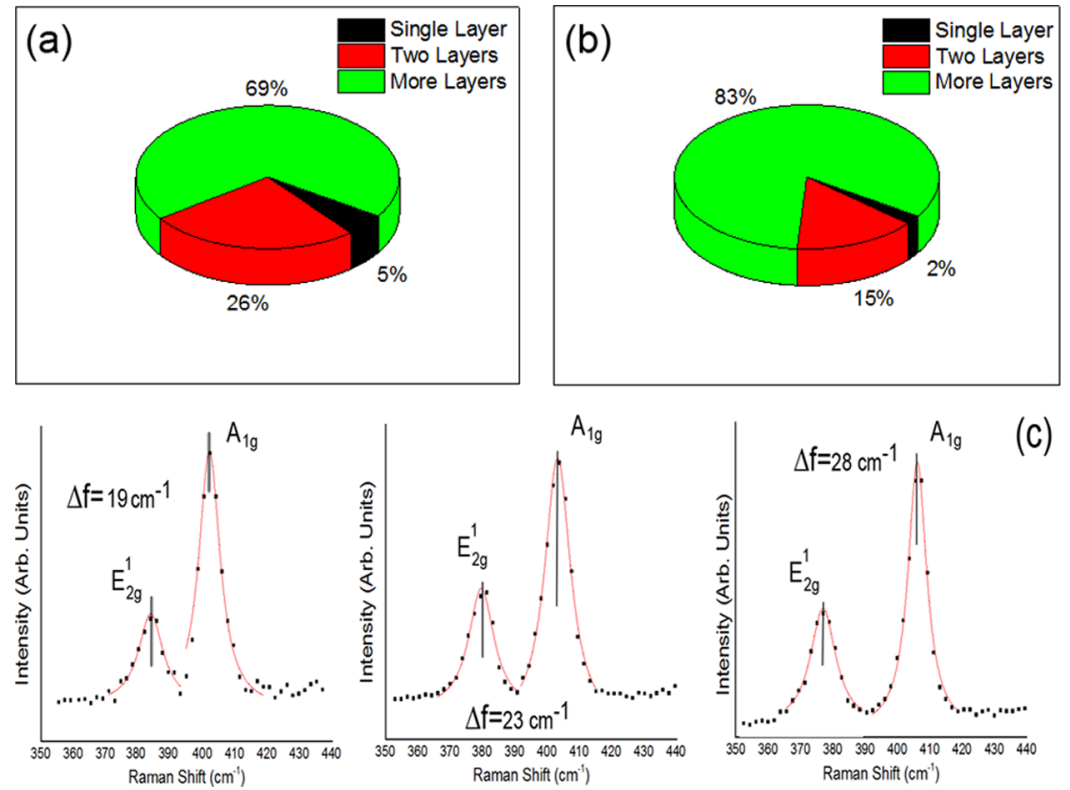

Figure 4. Statistical analyses based on the Raman spectrum to evaluate the number of deposited $\mathrm{MoS}_{2}$ layers. (a) Metallic nanoholes deposited at 2 $\mathrm{V}$; (b) 3D nanoantennas deposited at $15 \mathrm{~V}$; (c) examples of analyzed spectra.
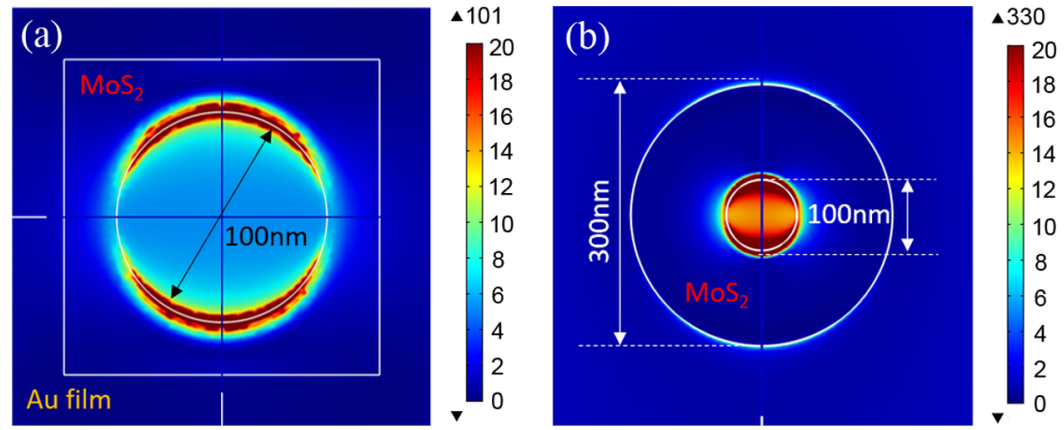

Figure 5. Finite element method (FEM) simulations of the investigated structures covered with one monolayer of $\mathrm{MoS}_{2}$ (simulations performed at excitation wavelength $532 \mathrm{~nm}$ ). In both cases, the field maps refer to the interface between metal and $\mathrm{MoS}_{2}$. (a) Top view of the EM field intensity $\left(\left|E / E_{0}\right|^{2}\right)$ in the case of the nanohole on a metallic (Au) film covered with a $\mathrm{MoS}_{2}$ layer. (b) Top view of $\left|E / E_{0}\right|^{2}$ in the case of $3 \mathrm{D}$ Au antenna covered with a $\mathrm{MoS}_{2}$ layer. In both cases, the $\mathrm{MoS}_{2}$ layer covers a $100 \mathrm{~nm}$ large hole.

this parameter can be a reliable quantity to count the number of layers of $\mathrm{MoS}_{2}$. We used $\Delta f$ to evaluate the percentage of single, double, or more layers of flakes deposited on the considered array. We considered " 1 layer" to have a $\Delta f$ equal to $18 \mathrm{~cm}^{-1}$, "2 layers" when for a value of $22 \mathrm{~cm}^{-1}$ and "more layers" when it is above $23 \mathrm{~cm}^{-1}$.

From our analysis, we can conclude that, while it does not seem possible to achieve single layer deposition with the higher applied potential (15 and $25 \mathrm{~V}$ for planar and 3D nanoholes, respectively), this is possible for a lower applied voltage $(2 \mathrm{~V}$ for planar nanoholes and $15 \mathrm{~V}$ for 3D antennas). In the case of metallic nanoholes (Figure 4a), a single layer can be deposited in about $5 \%$ of the cases, while the major amount of the deposited flakes is either double $(26 \%)$ or more than two layers (Figure $4 \mathrm{~b}$ ). For the 3D nanoantennas where, due to the high voltage needed for the deposition, single-layer flakes were only deposited rarely, while the majority of them resulted in two or more deposited layers. It should be mentioned that the evaluation of deposited flakes' thickness can be in principle performed by means of atomic force microscopy (AFM). This method, in our case, is less reliable with respect to Raman mapping not only due to the high time-cost of the measurements but also because the roughness of our metallic layer is comparable to a single-layer thickness. As an example, we have reported (see SI\#6) an AFM map showing the presence of a single layer.

Finally, statistical analyses based on Raman maps are used to also give an estimation of the plasmonic field enhancement mentioned previously. For this approximation, we designed the experimental evaluation as the following. It is not possible to control the size of the flakes deposited over the array, but it is well-known that the deposited flakes play a role in the intensity of the Raman peaks. An evaluation of the plasmonic field enhancement can therefore be reasonably obtained with a statistical comparison (over an array of about 200 different flakes) between the signals from plasmonic and nonplasmonic hole arrays. We performed similar EPD on both gold and $\mathrm{Si}_{3} \mathrm{~N}_{4}$ nanoholes and the mean intensities of the out-of-plane $\mathrm{A}_{1 \mathrm{~g}}$ peak over the whole array were considered for all of the cases. The ratio between these values allows us to estimate a plasmonic enhancement of approximately 5 and 9 for the metallic nanoholes and 3D plasmonic antennas, respectively (see SI\#7). 
Computer simulations (see Methods for details) can be used to better evaluate the observed enhancement effect. $^{34}$ In particular, we consider the two structures investigated, i.e., nanoholes and 3D antennas. Figure 5 illustrates the obtained field intensity confinement/enhancement that can be achieved in the two cases into the $2 \mathrm{D}$ layer. As can be easily seen in the case of a $\mathrm{MoS}_{2}$ flake deposited on top of a 3D antenna (Figure $5 b)$ the electromagnetic field intensity is more confined and can reach higher values with respect to the case of a nanohole on a metallic film (Figure 2a). This is also confirmed in our experiments.

\section{CONCLUSIONS}

In summary, here, we have presented a proof-of-concept experiment on a new scheme for the EPD of $2 \mathrm{D}$ materials on nanoholes. Our results demonstrate that whilst it is always possible to achieve a site-selective deposition of $\mathrm{MoS}_{2}$ flakes and single-layer flakes can be deposited, the process, in terms of the number of deposited layers, is hard to control. An overview of the whole deposition procedure can be surmised as follows. The applied potential creates an electroosmotic flow from one side of the chamber to the other, and the charged flakes move along the flow. The nanosized holes on the substrate act as a filter and the particles are trapped at the locations of the holes until the holes are completely clogged. Therefore, how many flakes are deposited depends on their starting shape and size. Moreover, it also depends on the applied voltage and reasonably from the used solvents. We reason that better control of the process can be achieved by altering the EPD conditions and the exfoliation procedure to obtain better-quality, single-layer flakes in solution. In this work, the aim was to obtain stable colloids of a few layers of $\mathrm{MoS}_{2}$ without the use of surfactants, which could affect the deposition process. Therefore, a chemical exfoliation approach was pursued, since it can provide highly exfoliated nanosheets with negative charges on the surface. ${ }^{52}$ However, due to the harshness of this process, $\mathrm{MoS}_{2}$ flakes can be crashed, causing the broadening of the size dispersion. Our process was found to be effective for all of the flakes' sizes, however, being EPD, a process influenced by hydrodynamical behavior of the material, a narrower distribution of lateral size and thickness would allow a sharper control on the deposition parameters. Hence, we believe that improving the synthesis procedure could result in an enhancement of the plasmonic response of these systems as well. Moreover, it is worthwhile to note that our strategy to obtain $\mathrm{MoS}_{2}$ nanosheets is rather slow, but the throughput of the Li-based exfoliation can be increased dramatically by switching from chemical to electrochemical intercalation. ${ }^{53,54}$ In this regard, the preparation of single-layer $\mathrm{MoS}_{2}$ would then be quick enough to make feasible the application of these systems on large scales.

Regardless, we have demonstrated that this fabrication procedure can be used to prepare hybrid plasmonic nanostructures over large arrays. This procedure is low-cost and does not require the use of complex lithographic processes. This strategy can be applied to many $2 \mathrm{D}$ materials for which the net charge of their surface can be used to guide the electrophoretic process. We believe that such an approach can find interesting applications in new hybrid devices for photoluminescence, strong coupling, and valley polarization studies.

\section{METHODS}

Exfoliation of $\mathrm{MoS}_{2}$. In a glove-box (water $<1 \mathrm{ppm}, \mathrm{O}_{2}<$ $10 \mathrm{ppm}), \mathrm{LiBH}_{4}(0.109 \mathrm{~g}, 5 \mathrm{mmol})$ and $\mathrm{MoS}_{2}(0.320 \mathrm{~g}, 2$ $\mathrm{mmol}$ ) were ground in a mortar and then transferred to a Schlenk-tube. This was then brought outside the glove-box and connected to a Schlenk-line. The mixture was then heated in a sand bath at $330{ }^{\circ} \mathrm{C}$ for 4 days under nitrogen. Afterward, the Schlenk-tube was again returned to dry-box, where it was ground with additional $\mathrm{LiBH}_{4}(0.109 \mathrm{~g}, 5 \mathrm{mmol})$. The sample was subsequently heated for another 3 days at $330{ }^{\circ} \mathrm{C}$ under nitrogen.

The intercalation product was added in a single shot in 270 $\mathrm{mL}$ of degassed water and the resulting suspension was bathsonicated for $1 \mathrm{~h}$ to facilitate the exfoliation.

To remove the $\mathrm{LiOH}$ produced, the suspension was equally divided into six centrifugation tubes (45 mL/tube) and centrifuged at $10000 \mathrm{rpm}(23478 g)$ for $20 \mathrm{~min}$ for three times, replacing the supernatant with a clean solvent.

To select the flake size, the suspension purified by $\mathrm{LiOH}$, was progressively centrifuged at 8000 ( $15026 \mathrm{~g}-8 \mathrm{k}$ fraction), $6000(8452 g-6 \mathrm{k}), 4000(3757 g-4 \mathrm{k}), 3000(2113 g-3 \mathrm{k})$, $2000(939 g-2 \mathrm{k})$, and $1000 \mathrm{rpm}(235 g-1 \mathrm{k})$, collecting each time the top $2 / 3$ of the supernatant and replacing it with ultrapure water.

Fabrication of Plasmonic Nanostructures. The fabrication of the metallic nanoholes follows simple and robust procedures for the $2 \mathrm{D}$ and $3 \mathrm{D}$ geometry. In both cases, the substrate was a $\mathrm{Si}_{3} \mathrm{~N}_{4}$ membrane (100 nm thick) prepared on a Silicon chip. The $2 \mathrm{D}$ holes were prepared by means of FIB milling with a voltage of $30 \mathrm{keV}$ and a current of $80 \mathrm{pA}$. After milling, a thin layer of gold, ca. $100 \mathrm{~nm}$, was deposited on the top side of the membrane. The fabrication of the $3 \mathrm{D}$ nanoantennas array follows the procedure laid out in ref 24 . A thin layer of S1813 optical resist is spin coated on top of the membrane with a final thickness equal to the height of the structure we want to obtain. This layer is exposed by the secondary electron during the FIB milling of the nanoholes from the bottom of the membrane, afterward the resist is developed in acetone and a final metal deposition (ca. $40 \mathrm{~nm}$ ) produces the $3 \mathrm{D}$ hollow antenna array. For these experiments, $3 \mathrm{D}$ antennas with the inner diameter between 30 and $100 \mathrm{~nm}$ and final height between 1000 and $2000 \mathrm{~nm}$ were prepared.

FEM Simulation of $\mathrm{MoS}_{2}$ Flakes Integrated over Plasmonic Nanostructures. We investigate the plasmonic properties of the structure by means of finite element method (FEM) simulations using an RF module in Comsol Multiphysics taking into account the geometries that were actually fabricated. For the nanohole array, we consider a hole of 100 $\mathrm{nm}$ in diameter in a $100 \mathrm{~nm}$ thick $\mathrm{Si}_{3} \mathrm{~N}_{4}$ membrane with a 50 $\mathrm{nm}$ thick Au layer. In the case of the $3 \mathrm{D}$ antennas, we consider an antenna with a height equal to $1000 \mathrm{~nm}$ and an inner and outer diameter of 100 and $300 \mathrm{~nm}$, respectively. A top single layer of $\mathrm{MoS}_{2}$ has been considered for both cases.

The refractive indices of $\mathrm{Au}$ and $\mathrm{MoS}_{2}$ were taken from the works of Rakic et al. and Zhang et al. ${ }^{55,56}$ The model computes the electromagnetic field distribution in each point of the simulation region, enabling the extraction of the quantities plotted in Figure 5. A single nanostructure was considered by setting the unit cell to be $400 \mathrm{~nm}$ wide in both the $x$ - and $y$ directions, with perfect matching layers $(150 \mathrm{~nm}$ thick) at the borders. A linearly polarized incident plane wave was assumed to impinge on the structure from the air side. 
Measurement of Dynamics Light Scattering of $\mathrm{MoS}_{2}$ Flakes. DLS experiments were performed using a Malvern Zetasizer nano ZS and the measurements were evaluated using Zetasizer software. Since polar solvents have been used in this study, 1.5 was chosen as the value for $f(k a)$ (Smoluchowski approximation). Thanks to this approximation, the influence of the size of the material in suspension is limited, allowing the use of Henry's equation (therefore DLS) for the evaluation of electrophoretic mobility of nonspherical materials. ${ }^{57,58}$ Data are reported as the average of three measurements $(n=3) \pm$ SD. Samples were measured at $25{ }^{\circ} \mathrm{C}$ in disposable folded capillary cells (DTS1070) in water dispersants.

\section{ASSOCIATED CONTENT}

\section{S Supporting Information}

The Supporting Information is available free of charge on the ACS Publications website at DOI: 10.1021/acsomega.9b00965.

Microfluidic chamber used for EPD (Figure S1); example of drop casting on $3 \mathrm{D}$ antennas (Figure S2); investigation on $\mathrm{MoS}_{2}$ degradation by means of $\zeta$ potential (Supporting Note 3, Tables S1 and S2); example of electrophoretic deposition on tall 3D antennas (Figure S3); example of EPD on $\mathrm{Si}_{3} \mathrm{~N}_{4}$ pores (Figure S4); Raman maps reporting shifts between 400 and $410 \mathrm{~cm}^{-1}$ over an array of $\mathrm{MoS}_{2}$ flakes deposited onto $\mathrm{Si}_{3} \mathrm{~N}_{4}$ holes (Figure S5); example of AFM maps on $\mathrm{MoS}_{2}$ flakes (Figure S6); estimation of plasmonic enhancement from Raman maps (Figure S7) (PDF)

\section{AUTHOR INFORMATION}

\section{Corresponding Author}

*E-mail: denis.garoli@iit.it.

\section{ORCID $\odot$}

Giorgia Giovannini: 0000-0003-4496-3709

Michele Serri: 0000-0002-6018-5284

Michele Dipalo: 0000-0002-1823-8231

Stefano Agnoli: 0000-0001-5204-5460

Denis Garoli: 0000-0002-5418-7494

\section{Author Contributions}

D.M. worked on the $\mathrm{MoS}_{2}$ preparation and characterization; A.J. and G.G. performed the electrophoretic deposition; P.P. and M.S. fabricated and characterized the structures; G.G. performed DLS characterization; N.M. performed the simulations; M.D. and D.D. helped during the manuscript preparation; P.V., S.A., and F.D.A. helped in the discussion and D.G. conceived the EPD method and coordinated the work.

\section{Notes}

The authors declare no competing financial interest.

\section{ACKNOWLEDGMENTS}

We thank the Materials Characterization Facility at the Fondazione Istituto Italiano di Tecnologia for help with AFM. The research leading to these results has received funding from the Horizon 2020 Program, FET-Open: PROSEQO, Grant Agreement n. [687089].

\section{REFERENCES}

(1) Geim, A. K.; Novoselov, K. S. The Rise of Graphene. Nat. Mater. 2007, 6, 183-191.
(2) Cooper, D. R.; D’Anjou, B.; Ghattamaneni, N.; Harack, B.; Hilke, M.; Horth, A.; Majlis, N.; Massicotte, M.; Vandsburger, L.; Whiteway, E.; et al. Experimental Review of Graphene. ISRN Condens. Matter Phys. 2012, 2012, 1-56.

(3) Mas-Ballesté, R.; Gómez-Navarro, C.; Gómez-Herrero, J.; Zamora, F. 2D Materials: To Graphene and Beyond. Nanoscale 2011, 3, 20-30.

(4) Agnoli, S.; Granozzi, G. Second Generation Graphene: Opportunities and Challenges for Surface Science. Surf. Sci. 2013, $609,1-5$.

(5) Chhowalla, M.; Shin, H. S.; Eda, G.; Li, L. J.; Loh, K. P.; Zhang, H. The Chemistry of Two-Dimensional Layered Transition Metal Dichalcogenide Nanosheets. Nat. Chem. 2013, 5, 263-275.

(6) Wang, Q. H.; Kalantar-Zadeh, K.; Kis, A.; Coleman, J. N.; Strano, M. S. Electronics and Optoelectronics of Two-Dimensional Transition Metal Dichalcogenides. Nat. Nanotechnol. 2012, 7, 699712 .

(7) Mak, K. F.; Lee, C.; Hone, J.; Shan, J.; Heinz, T. F. Atomically Thin $\mathrm{MoS}_{2}$ : A New Direct-Gap Semiconductor. Phys. Rev. Lett. 2010, $105,2-5$.

(8) Prins, F.; Goodman, A. J.; Tisdale, W. A. Reduced Dielectric Screening and Enhanced Energy Transfer in Single- and Few-Layer $\mathrm{MoS}_{2}$. Nano Lett. 2014, 14, 6087-6091.

(9) Goodfellow, K. M.; Beams, R.; Chakraborty, C.; Novotny, L.; Vamivakas, A. N. Integrated Nanophotonics Based on Nanowire Plasmons and Atomically Thin Material. Optica 2014, 1, 149-152.

(10) Lee, H. S.; Kim, M. S.; Jin, Y.; Han, G. H.; Lee, Y. H.; Kim, J. Selective Amplification of the Primary Exciton in a $\mathrm{MoS}_{2}$ Monolayer. Phys. Rev. Lett. 2015, 115, No. 226801.

(11) Geim, A. K.; Grigorieva, I. V. Van Der Waals Heterostructures. Nature 2013, 499, 419-425.

(12) Rigosi, A. F.; Hill, H. M.; Li, Y.; Chernikov, A.; Heinz, T. F. Probing Interlayer Interactions in Transition Metal Dichalcogenide Heterostructures by Optical Spectroscopy: $\mathrm{MoS}_{2} / \mathrm{WS}_{2}$ and $\mathrm{MoSe}_{2} /$ $\mathrm{WSe}_{2}$. Nano Lett. 2015, 15, 5033-5038.

(13) Kang, Y.; Najmaei, S.; Liu, Z.; Bao, Y.; Wang, Y.; Zhu, X.; Halas, N. J.; Nordlander, P.; Ajayan, P. M.; Lou, J.; et al. Plasmonic Hot Electron Induced Structural Phase Transition in a $\mathrm{MoS}_{2}$ Monolayer. Adv. Mater. 2014, 26, 6467-6471.

(14) Zhan, Y.; Liu, Z.; Najmaei, S.; Ajayan, P. M.; Lou, J. Large Area Vapor Phase Growth and Characterization of $\mathrm{MoS}_{2}$ Atomic Layers on $\mathrm{SiO}_{2}$ Substrate. Small 2012, 8, 966-971.

(15) Li, Z.; Ye, R.; Feng, R.; Kang, Y.; Zhu, X.; Tour, J. M.; Fang, Z. Graphene Quantum Dots Doping of $\mathrm{MoS}_{2}$ Monolayers. Adv. Mater. 2015, 27, 5235-5240.

(16) Chen, Y. J.; Cain, J. D.; Stanev, T. K.; Dravid, V. P.; Stern, N. P. Valley-Polarized Exciton-Polaritons in a Monolayer Semiconductor. Nat. Photonics 2017, 11, 431-435.

(17) Liu, W.; Lee, B.; Naylor, C. H.; Ee, H. S.; Park, J.; Johnson, A. T. C.; Agarwal, R. Strong Exciton-Plasmon Coupling in $\mathrm{MoS}_{2}$ Coupled with Plasmonic Lattice. Nano Lett. 2016, 16, 1262-1269.

(18) Yang, W.; Shang, J.; Wang, J.; Shen, X.; Cao, B.; Peimyoo, N.; Zou, C.; Chen, Y.; Wang, Y.; Cong, C.; et al. Electrically Tunable Valley-Light Emitting Diode (VLED) Based on CVD-Grown Monolayer $\mathrm{WS}_{2}$. Nano Lett. 2016, 16, 1560-1567.

(19) Wu, S.; Buckley, S.; Schaibley, J. R.; Feng, L.; Yan, J.; Mandrus, D. G.; Hatami, F.; Yao, W.; Vučković, J.; Majumdar, A.; et al. Monolayer Semiconductor Nanocavity Lasers with Ultralow Thresholds. Nature 2015, 520, 69-72.

(20) Liu, X.; Galfsky, T.; Sun, Z.; Xia, F.; Lin, E. C.; Lee, Y. H.; Kéna-Cohen, S.; Menon, V. M. Strong Light-Matter Coupling in Two-Dimensional Atomic Crystals. Nat. Photonics 2015, 9, 30-34.

(21) Butun, S.; Tongay, S.; Aydin, K. Enhanced Light Emission from Large-Area Monolayer $\mathrm{MoS}_{2}$ Using Plasmonic Nanodisc Arrays. Nano Lett. 2015, 15, 2700-2704.

(22) Lee, B.; Park, J.; Han, G. H.; Ee, H.; Naylor, C. H.; Liu, W.; Johnson, A. T. C.; Agarwal, R. Fano Resonance and Spectrally Modified Photoluminescence Enhancement in Monolayer $\mathrm{MoS}_{2}$ 
Integrated with Plasmonic Nanoantenna Array. Nano Lett. 2015, 15, 3646-3653.

(23) Yang, A.; Li, Z.; Knudson, M. P.; Hryn, A. J.; Wang, W.; Aydin, K.; Odom, T. W. Unidirectional Lasing from Template-Stripped TwoDimensional Plasmonic Crystals. ACS Nano 2015, 9, 11582-11588.

(24) Garoli, D.; Mosconi, D.; Miele, E.; Maccaferri, N.; Ardini, M.; Giovannini, G.; Di Palo, M.; Agnoli, S.; De Angelis, F. Hybrid Plasmonic Nanostructures Base on Controlled Integration of MoS2 Flakes on Metallic Nanoholes. Nanoscale 2018, 10, 17105-17111.

(25) Chen, H.; Yang, J.; Rusak, E.; Straubel, J.; Guo, R.; Myint, Y. W.; Pei, J.; Decker, M.; Staude, I.; Rockstuhl, C.; et al. Manipulation of Photoluminescence of Two-Dimensional $\mathrm{MoSe}_{2}$ by Gold Nanoantennas. Sci. Rep. 2016, 6, No. 22296

(26) Li, Z.; Li, Y.; Han, T.; Wang, X.; Yu, Y.; Tay, B.; Liu, Z.; Fang, Z. Tailoring $\mathrm{MoS}_{2}$ Exciton-Plasmon Interaction by Optical Spin-Orbit Coupling. ACS Nano 2017, 11, 1165-1171.

(27) Huang, J.; Akselrod, G. M.; Ming, T.; Kong, J.; Mikkelsen, M. H. Tailored Emission Spectrum of 2D Semiconductors Using Plasmonic Nanocavities. ACS Photonics 2018, 5, 552-558.

(28) Janisch, C.; Song, H.; Zhou, C.; Lin, Z.; Elías, A. L.; Ji, D.; Terrones, M.; Gan, Q.; Liu, Z. $\mathrm{MoS}_{2}$ Monolayers on Nanocavities: Enhancement in Light-matter Interaction. 2D Mater. 2016, 3, No. 025017.

(29) Wang, Z.; Dong, Z.; Gu, Y.; Chang, Y. H.; Zhang, L.; Li, L. J.; Zhao, W.; Eda, G.; Zhang, W.; Grinblat, G.; et al. Giant Photoluminescence Enhancement in Tungsten-Diselenide-Gold Plasmonic Hybrid Structures. Nat. Commun. 2016, 7, No. 11283.

(30) Mukherjee, B.; Kaushik, N.; Tripathi, R. P. N.; Joseph, A. M.; Mohapatra, P. K.; Dhar, S.; Singh, B. P.; Kumar, G. V. P.; Simsek, E.; Lodha, S. Exciton Emission Intensity Modulation of Monolayer $\mathrm{MoS}_{2}$ via Au Plasmon Coupling. Sci. Rep. 2017, 7, No. 41175.

(31) Yu, Y.; Ji, Z.; Zu, S.; Du, B.; Kang, Y.; Li, Z.; Zhou, Z.; Shi, K.; Fang, Z. Ultrafast Plasmonic Hot Electron Transfer in Au Nanoantenna $/ \mathrm{MoS}_{2}$ Heterostructures. Adv. Funct. Mater. 2016, 26, 63946401

(32) Kang, Y.; Gong, Y.; Hu, Z.; Li, Z.; Qiu, Z.; Zhu, X.; Ajayan, P. M.; Fang, Z. Plasmonic Hot Electron Enhanced $\mathrm{MoS}_{2}$ Photocatalysis in Hydrogen Evolution. Nanoscale 2015, 7, 4482-4488.

(33) Zhao, Y.; Tang, M.; Liao, Q.; Li, Z.; Li, H.; Xi, K.; Tan, L.; Zhang, M.; Xu, D.; Chen, H. Y. Disposable $\mathrm{MoS}_{2}$-Arrayed MALDI MS Chip for High-Throughput and Rapid Quantification of Sulfonamides in Multiple Real Samples. ACS Sens. 2018, 3, 806-814.

(34) Srinivasan, K.; Subramanian, K.; Murugan, K.; Dinakaran, K. Sensitive Fluorescence Detection of Mercury (II) in Aqueous Solution by the Fluorescence Quenching Effect of $\mathrm{MoS}_{2}$ with DNA Functionalized Carbon Dots. Analyst 2016, 141, 6344-6352.

(35) Lin, J.; Li, H.; Zhang, H.; Chen, W. Plasmonic Enhancement of Photocurrent in $\mathrm{MoS}_{2}$ Field-Effect-Transistor. Appl. Phys. Lett. 2013, 102, No. 203109.

(36) Miao, J.; Hu, W.; Jing, Y.; Luo, W.; Liao, L.; Pan, A.; Wu, S.; Cheng, J.; Chen, X.; Lu, W. Surface Plasmon-Enhanced Photodetection in Few Layer $\mathrm{MoS}_{2}$ Phototransistors with Au Nanostructure Arrays. Small 2015, 11, 2392-2398.

(37) Feng, J.; Graf, M.; Liu, K.; Ovchinnikov, D.; Dumcenco, D.; Heiranian, M.; Nandigana, V.; Aluru, N. R.; Kis, A.; Radenovic, A. Single-Layer $\mathrm{MoS}_{2}$ Nanopores as Nanopower Generators. Nature 2016, 536, 197-200.

(38) Zhu, C.; Zeng, Z.; Li, H.; Li, F.; Fan, C.; Zhang, H. SingleLayer $\mathrm{MoS}_{2}$-Based Nanoprobes for Homogeneous Detection of Biomolecules. J. Am. Chem. Soc. 2013, 135, 5998-6001.

(39) Aruna, S. T.; Rajam, K. S. A Study on the Electrophoretic Deposition of 8YSZ Coating Using Mixture of Acetone and Ethanol Solvents. Mater. Chem. Phys. 2008, 111, 131-136.

(40) Jian, G.; Lu, S.; Zhou, D.; Yang, J.; Fu, Q. Cobalt Ferrite Dispersion in Organic Solvents for Electrophoretic Deposition: Influence of Suspension Parameters on the Film Microstructure. Mater. Chem. Phys. 2014, 143, 653-660.

(41) Khanali, O.; Rajabi, M.; Baghshahi, S. Effect of Non-Aqueous Solvents on Deposition Properties in Electrophoretic Deposition
Process of Yttria Stabilized Zirconia Nano-Powders. J. Ceram. Process. Res. 2017, 18, 735-742.

(42) Nam, M. S.; Patil, U.; Park, B.; Sim, H. B.; Jun, S. C. A Binder Free Synthesis of 1D PANI and 2D $\mathrm{MoS}_{2}$ Nanostructured Hybrid Composite Electrodes by the Electrophoretic Deposition (EPD) Method for Supercapacitor Application. RSC Adv. 2016, 6, 101592101601.

(43) Liu, Y.; Ren, L.; Zhang, Z.; Qi, X.; Li, H.; Zhong, J. 3D BinderFree $\mathrm{MoSe}_{2}$ Nanosheets/Carbon Cloth Electrodes for Efficient and Stable Hydrogen Evolution Prepared by Simple Electrophoresis Deposition Strategy. Sci. Rep. 2016, 6, No. 22516.

(44) Lin, J.-Y.; Chan, C.-Y.; Chou, S.-W. Electrophoretic Deposition of Transparent $\mathrm{MoS}_{2}-$ graphene Nanosheet Composite Films as Counter Electrodes in Dye-Sensitized Solar Cells. Chem. Commun. 2013, 49, 1440.

(45) Garoli, D.; Zilio, P.; Gorodetski, Y.; Tantussi, F.; De Angelis, F. Beaming of Helical Light from Plasmonic Vortices via Adiabatically Tapered Nanotip. Nano Lett. 2016, 16, 6636-6643.

(46) Garoli, D.; Calandrini, E.; Bozzola, A.; Ortolani, M.; Cattarin, S.; Barison, S.; Toma, A.; De Angelis, F. Boosting Infrared Energy Transfer in 3D Nanoporous Gold Antennas. Nanoscale 2017, 9, 915922.

(47) Garoli, D.; Della Giustina, G. Directly Patternable High Refractive Index Ferroelectric Sol-Gel Resist. Mater. Chem. Phys. 2015, 164, 63-70.

(48) Cooper, R. C.; Lee, C.; Marianetti, C. A.; Wei, X.; Hone, J.; Kysar, J. W. Nonlinear Elastic Behavior of Two-Dimensional Molybdenum Disulfide. Phys. Rev. B 2013, 87, No. 035423.

(49) Castellanos-Gomez, A.; Agrat, N.; Rubio-Bollinger, G. Optical Identification of Atomically Thin Dichalcogenide Crystals. Appl. Phys. Lett. 2010, 96, No. 213116

(50) Splendiani, A.; Sun, L.; Zhang, Y.; Li, T.; Kim, J.; Chim, C. Y.; Galli, G.; Wang, F. Emerging Photoluminescence in Monolayer $\mathrm{MoS}_{2}$. Nano Lett. 2010, 10, 1271-1275.

(51) Lee, C.; Yan, H.; Brus, L.; Heinz, T.; Hone, J.; Ryu, S. Anomalous Lattice Vibrations of Single-and Few-Layer $\mathrm{MoS}_{2}$. ACS Nano 2010, 4, 2695-2700.

(52) Eda, G.; Yamaguchi, H.; Voiry, D.; Fujita, T.; Chen, M.; Chhowalla, M. Photoluminescence from Chemically Exfoliated $\mathrm{MoS}_{2}$. Nano Lett. 2011, 11, 5111-5116.

(53) Zeng, Z.; Yin, Z.; Huang, X.; Li, H.; He, Q.; Lu, G.; Boey, F.; Zhang, H. Single-Layer Semiconducting Nanosheets: High-Yield Preparation and Device Fabrication. Angew. Chem., Int. Ed. 2011, 50, 11093-11097.

(54) Benavente, E.; Santa Ana, M. A.; Mendizábal, F.; González, G. Intercalation Chemistry of Molybdenum Disulfide. Coord. Chem. Rev. 2002, 224, 87-109.

(55) Rakic, A. D.; Djurisic, A. B.; Elazar, J. M.; Majewski, M. L. Optical Properties of Metallic Films for Vertical-Cavity Optoelectronic Devices. Appl. Opt. 1998, 37, 5271-5283.

(56) Zhang, H.; Ma, Y.; Wan, Y.; Rong, X.; Xie, Z.; Wang, W.; Dai, L. Measuring the Refractive Index of Highly Crystalline Monolayer $\mathrm{MoS}_{2}$ with High Confidence. Sci. Rep. 2015, 5, No. 8440.

(57) Lee, V.; Whittaker, L.; Yaye, C.; Baroudi, K. M.; Fischer, D. A.; Banerjee, S. Large-Area Chemically Modified Graphene Films: Electrophoretic Deposition and Characterization by Soft X-ray Absorption Spectroscopy. Chem. Mater. 2009, 21, 3905-3916.

(58) Jastrzębska, A. M.; Karcz, J.; Letmanowski, R.; Zabost, D.; Ciecierska, E.; Zdunek, J.; Karwowska, E.; Siekierski, M.; Olszyna, A.; Kunicki, A. Synthesis of the RGO/Al2O3 core-shell nanocomposite flakes and characterization of their unique electrostatic properties using zeta potential measurements. Appl. Surf. Sci. 2016, 362, 577594. 